\title{
Comparison between one-dimensional and two-dimensional models for Josephson junctions of overlap type
}

\author{
Eilbeck, J. C; Lomdahl, P.S.; Olsen, O.H.; Samuelsen, Mogens Rugholm
}

Published in:

Journal of Applied Physics

Link to article, DOI:

$10.1063 / 1.335478$

Publication date:

1985

Document Version

Publisher's PDF, also known as Version of record

Link back to DTU Orbit

Citation (APA):

Eilbeck, J. C., Lomdahl, P. S., Olsen, O. H., \& Samuelsen, M. R. (1985). Comparison between one-dimensional and two-dimensional models for Josephson junctions of overlap type. Journal of Applied Physics, 57(3), 861866. https://doi.org/10.1063/1.335478

\section{General rights}

Copyright and moral rights for the publications made accessible in the public portal are retained by the authors and/or other copyright owners and it is a condition of accessing publications that users recognise and abide by the legal requirements associated with these rights.

- Users may download and print one copy of any publication from the public portal for the purpose of private study or research.

- You may not further distribute the material or use it for any profit-making activity or commercial gain

- You may freely distribute the URL identifying the publication in the public portal 


\title{
Comparison between one-dimensional and two-dimensional models for Josephson Junctions of overlap type
}

\author{
J. C. Eilbeck, ${ }^{\text {a) }}$ P. S. Lomdahi, and O. H. Olsen ${ }^{\text {b) }}$ \\ Center for Nonlinear Studies, Los Alamos National Laboratory, Los Alamos, New Mexico 87545 \\ M. R. Samuelsen \\ Physics Laboratory I, The Technical University of Denmark, DK-2800 Lyngby, Denmark
}

(Received 22 June 1984; accepted for publication 24 July 1984)

\begin{abstract}
A two-dimensional model of Josephson junction of overlap type is presented. The energy input is provided through induced magnetic fields modeled by a set of boundary conditions. In the limit of a very narrow junction, this model reduces to the one-dimensional model. Further, an equation derived for the critical current leads in this limit to the critical current obtained from the onedimensional model. Comparisons between stationary fluxon velocities obtained from the two models by means of numerical computations show that the difference is negligible. This supports the experimental observation that measurements are insensitive to the width of the junction.
\end{abstract}

\section{INTRODUCTION}

The topic of fuxons in long Josephson junctions has attracted considerable interest in recent years. There are several reasons for this. First, the Josephson junction is a wellunderstood solid-state system on which experimental studies are undertaken with relative ease. Secondly, there have been suggestions for applications within such diverse fields as microwave oscillators/amplifiers and data processing systems.

Fluxon dynamics in long Josephson junctions have mainly been investigated theoretically by means of analytical methods, ${ }^{1-2}$ perturbation analysis, ${ }^{3-5}$ and numerical simulations. $^{6-8}$ These studies have been made only in the framework of the model described by the one-dimensional sine-Gordon equation, which is based on the assumption that the width of the junction is much smaller than the Josephson penetration depth $\lambda_{J}$. The results from this model agree almost perfectly with the experimental findings.

In Ref. 6, a detailed numerical study of a one-dimensional sine-Gordon model of the Josephson tunnel junction was compared with experimental measurements on the junction. Although the width of the junction was not negligible, a good agreement was found for the current-voltage characteristic, power output, and for the shape and height of the zero-field steps. In Ref. 9, results from the perturbation theory for fluxon motion in the modified one-dimensional sineGordon equation were compared with the experiments on long Josephson junctions with small damping constants. Again, the theory agrees almost perfectly with the experiments even though the width of the junction is comparable with $\lambda_{J}$.

The purpose of the present paper is to present a twodimensional model of the Josephson junction of overlap type. When the width of the junction is much smaller than $\lambda_{J}$ this model is shown to reduce to the one-dimensional

\footnotetext{
a) Permanent address: Department of Mathematics, Heriot-Watt University, Riccarton, Edinburgh EH14 4AS, UK.

b) Permanent address: Niro Atomizer, Systems Engineering, Gladsaxevej 305, DK-2860, Soeborg, Denmark.
}

model. For the two-dimensional model an equation for the critical current is derived. In the limit of a very narrow junction the critical current equals the critical current obtained from the one-dimensional model. Further comparisons between stationary fluxon velocities obtained from the two models by means of numerical computations show that even if the width is much larger then $\lambda_{J}$ the difference is negligible. This result supports the observation that experimental results are insensitive to the width of the junction.

The paper is organized as follows: in Sec. II, the twodimensional model is developed resulting in a perturbed sine-Gordon equation in two spatial dimensions-the energy input is provided through boundary conditions modeling the induced magnetic fields. This model is examined numerically in Sec. III and compared to the corresponding onedimensional model.

\section{MODEL EQUATION AND BOUNDARY CONDITIONS}

An overlap geometry Josephson tunnel junction consists of two superconducting metal layers separated by a thin insulating oxide layer of uniform which is small enough to permit quantum mechanical tunneling of electrons. The geometry is shown in Fig. 1. The tunneling supercurrent is described by the two basic Josephson equations

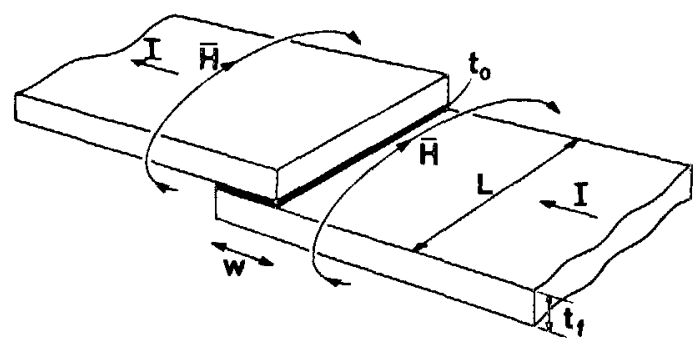

$$
\begin{aligned}
& t_{0} \ll \lambda_{L} \ll t_{1} \ll w \ll A_{S}<L \\
& 10^{-9} 5 \times 10^{-8} 2 \times 10^{-7} 5 \times 10^{-5} 2 \times 10^{-4} 10^{-3}[\mathrm{~m}]
\end{aligned}
$$

FIG. 1. Josephson tunnel juncton of overlap type. 


$$
\begin{aligned}
& j=j_{J} \sin \varphi, \\
& \frac{\partial \varphi}{\partial t}=\frac{2 e}{\hbar} V,
\end{aligned}
$$

where $\varphi=\varphi(x, y, t)$ is the difference between the phases of the order parameters of the two superconductors and $j=j$ $(x, y, t)$ is the Josephson current crossing the insulating layer per unit area, $j_{J}$ being the maximum supercurrent density. The voltage across the insulating barrier is given by $V=V$ $(x, y, t)$, and the constants $e$ and $\hbar$ are the charge of the electron and Planck's constant divided by $2 \pi$, respectively. The surface current density $i=i(x, y, t)$ is given by

$$
\mathbf{i}=-\hat{\mathbf{H}}=-\frac{1}{\mu_{0}} \widehat{\left(B_{x}, B_{y}\right)}=\frac{\hbar}{2 e d \mu_{0}} \nabla \varphi .
$$

Here $d=2 \lambda_{L}+t_{0}$ is the magnetic thickness of the junction, $\lambda_{L}$ is the London penetration depth, $\mu_{0}$ is the permeability of free space, and $B_{x}\left(B_{y}\right)$ is the $x$ component ( $y$ component) of B. The caret indicates an orthogonal vector.

The current density through the oxide layer is given by

$$
j_{z}=j_{J} \sin \varphi+\frac{\hbar}{2 e R} \varphi_{t}+\frac{\hbar C}{2 e} \varphi_{n},
$$

the second term on the right-hand side represents dissipative effects due to quasi-particle tunneling, $R$ being an effective normal resistance. The third term represents the energy stored in the barrier (i.e., a displacement current). Equation (3) is in fact just the resistivity-shunted-junction RSJ equation expressed in second order for a distributed tunneling area.

From now on, all the lengths $x, y, t_{0}, \lambda_{L}, t_{f}, w$, and $L$ are measured in units of the Josephson penetration depth $\lambda_{J}=\left(\hbar / 2 \mu_{0} e d j_{J}\right)^{1 / 2}$ and time $t$ in units of the reciprocal plasma $\omega_{p}^{-1}$, where $\omega_{p}=\left(2 e j_{J} / \hbar C\right)^{1 / 2}$. Finally, the equation of continuity

$$
\nabla \cdot \mathbf{i}-j_{z}=0 \text {, }
$$

substituted in Eq. (3) and using Eq. (2), yields the two-dimensional sine-Gordon equation

$$
\varphi_{x x}+\varphi_{y y}=\sin \varphi+\varphi_{u}+\alpha \varphi_{t},
$$

where $\alpha=(\hbar / 2 e R) \omega_{p}=\left(1 / \sqrt{\beta_{C}}\right), \beta_{C}$ is the usual McCumber parameter.

If losses in the London penetration layer are taken into account, Eq. (2) is changed. The voltage across the barrier is proportional to $\varphi_{t}(\mathrm{Eq} .1)$; therefore, the electric field along the oxide layer is proportional to $\nabla \varphi_{\mathrm{r}}$. The surface current density is

$$
\mathbf{i}=\lambda_{J} j_{J} \nabla\left(\varphi+\kappa \varphi_{\mathrm{t}}\right) \text {. }
$$

Further losses due to quasi-particle pair interference current can be included by replacing $\alpha \varphi_{\text {, }}$ with $\alpha \varphi_{t}(1+\epsilon \cos \varphi)$. Introducing these corrections into the equation of continuity we get

$$
\begin{aligned}
\kappa\left(\varphi_{x x}+\varphi_{y y}\right)_{t}+ & \varphi_{x x}+\varphi_{y y} \\
& =\sin \varphi+\varphi_{r t}+\alpha(1+\epsilon \cos \varphi) \varphi_{r},
\end{aligned}
$$

which is the usual two-dimensional model of the Josephson junction. Energy input to the system is provided from the magnetic fields induced along the boundaries. In the following, we determine the boundary conditions and show how the two-dimensional equation is reduced to one dimension.
The magnetic field along $y= \pm(w / 2)$ is approximately given by

$$
H\left(x, \pm \frac{w}{2}, t\right)= \pm \frac{I}{2\left(L+t_{f} \lambda_{J} j_{J}\right.} \approx \pm \eta \frac{w}{2},
$$

or

$$
\varphi_{y}\left(x, \pm \frac{w}{2}, t\right)= \pm \eta \frac{w}{2}= \pm \beta,
$$

for $|x| \leqslant(L / 2)$, where

$$
\eta=\frac{I}{L w j_{J}}
$$

is the uniform bias current through the barrier. From Ampere's law it follows that

$$
H[ \pm(L / 2), y, t] \approx 0,
$$

or

$$
\varphi_{x}[ \pm(L / 2), y, t] \approx 0 .
$$

Thus in the two-dimensional case, the energy-providing mechanism is the magnetic field via the boundary condition in Eq. (8).

Now, a solution of the form

$$
\varphi(x, y, t)=\varphi_{1}(x, t)+\varphi_{2}(y), \quad \varphi_{2}<1,
$$

reduces Eqs. (7) and (8) to

$$
\begin{aligned}
\kappa \varphi_{1, x x t} & +\varphi_{1, x x}=\sin \varphi_{1} \\
& +\alpha\left(1+\epsilon \cos \varphi_{1}\right) \varphi_{1, t}-\eta+\varphi_{1, t l},
\end{aligned}
$$

which is the one-dimensional sine-Gordon equation if

$$
\varphi_{2}(y)=\frac{\eta y^{2}}{2}, \quad \frac{\eta}{2}\left(\frac{w}{2}\right)^{2}<1
$$

Thus for $\eta w^{2} / 8<1$, the overlap geometry junction can be modeled by the one-dimensional sine-Gordon equation. We remark that $\boldsymbol{\eta}$ in this approximation might depend on $x$.

Finally in this section we examine the magnitude of the critical current obtained from the two-dimensional model. For the one-dimensional model of overlap geometry junctions the critical current is given by $I_{c}=j_{j} w L$ or $\eta_{c}=1$. When $\eta$ exceeds this value the phase as a whole starts to increase. For the two-dimensional junction the critical current for $w>l$ is given by

$$
\beta_{c}=\eta_{c} \frac{w}{2}=2 \text { or } \eta_{c}=\frac{4}{w} \text {. }
$$

If $\beta$ exceds this critical value, fiuxons (antifluxons) will be generated at the boundary $y=w / 2(y=-w / 2)$. In order to determine the critical current for smaller values of $w$ we need only consider the junction in the $y$ direction and set $\alpha=0$. The solution to $\varphi_{y y}=\sin \varphi$ is given by

$$
\sin \varphi= \pm 2 \operatorname{sn}(k, y) d n(k, y),
$$

or

$$
\varphi_{y}= \pm 2 k \mathrm{cn}(k, y)
$$

where sn, $\mathrm{cn}$, and dn are Jacobian elliptic functions of order $k$ and argument $y$, and also $l \sqrt{2}<k<1$. Equation (15) represents a static solution to Eq. (7) independent of $x$. Now an expression for the current can be found: 


$$
\begin{aligned}
\eta(w / 2) & =2 k \operatorname{cn}[k, K(k)-(w / 2)] \\
& =2 k \sqrt{1-k^{2}} \frac{\operatorname{sn}[k,(w / 2)]}{\operatorname{dn}[k,(w / 2)]} .
\end{aligned}
$$

The critical current is then the maximum value of $\eta$ obtained by variation of $k$ for specified $w$. For $w>1$, the elliptic inte$\operatorname{gral} K(k)>1$, thus $k \approx 1$ and $c n \approx 1$ which gives $\eta_{c}=4 / w$ in agreement with $\mathrm{Eq}$. (14). In the other limit, $w<1$, we have $\mathrm{sn} \approx w / 2$ and $\mathrm{dn} \approx 1$ :

$$
\eta=2 k \sqrt{1-k^{2}}
$$

which has a maximum $\eta_{c}=1$ for $k=1 / \sqrt{2}$. In Ref. 10 the relation between the critical current and the width of the junction has been obtained by means of numerical simulations and agrees with the result above.

In the following sections, we solve Eq. (5) numerically, applying the boundary conditions of Eqs. (8) and (10) in order to study the behavior of the single fluxon. Further, we compare the results obtained by varying the width $w$ with the results obtained from the one-dimensional model.

\section{NUMERICAL RESULTS}

In order to study the behavior of a single fluxon in a Josephson junction of overlap geometry, we use the model presented in the previous section. We solve the initial value problem

$$
\begin{aligned}
& \varphi_{x x}+\varphi_{y y}-\varphi_{t t}=\sin \varphi+\alpha \varphi_{t}, \\
& \varphi_{x}\left( \pm \frac{L}{2}, y, t\right)=0, \\
& \varphi_{y}\left[x, \pm \frac{w}{2}, t\right]= \pm \eta \frac{w}{2}= \pm \beta, \\
& \varphi(x, y, 0)=F(x, y, 0), \\
& \varphi_{t}(x, y, 0)=F,(x, y, 0),
\end{aligned}
$$

where the boundary conditions model the effect of the induced magnetic fields and the initial conditions are given by

$$
\begin{aligned}
& F(x, y, t)= 4 \tan ^{-1} \exp \left\{-\left[\xi_{1}(\beta)+w / 2+y\right]\right\} \\
&+4 \tan ^{-1} \exp \left[\gamma(u)\left(x+p y+u_{0} t-x_{0}\right)\right] \\
&+4 \tan ^{-1} \exp \left\{-\left[\xi_{2}(\beta)-w / 2+y\right]\right\}(19) \\
& \xi_{1}(\beta)=\ln \left\{\frac{2}{\beta}\left[1+\left(1-\frac{\beta^{2}}{4}\right)^{1 / 2}\right]\right\} \\
& \xi_{2}(\beta)=\ln \left\{\frac{2}{\beta}\left[1-\left(1-\frac{\beta^{2}}{4}\right)^{1 / 2}\right]\right\} \\
& \gamma(u)=\left(1-u^{2}\right)^{-1 / 2} .
\end{aligned}
$$

Here the first and the last term in the expression for $F(x, y, t)$ represent parts of static fluxons (perpendicular to the short direction of the junction) such that the boundary conditions are satisfied and the energy in the junction for $t=0$ is minimized. The middle term represents a fluxon with initial location and velocity $\left(x_{0}, y\right)$ and $u_{0}$ respectively. The physical scaling of velocity is given by $\lambda_{J} \omega_{p}$. Further for $p=0$, the fluxon is initially perpendicular to the long direction of the junction.

In the numerical solution we have applied an approximation ${ }^{11-12}$ based on a stabilized leap-frog scheme extended
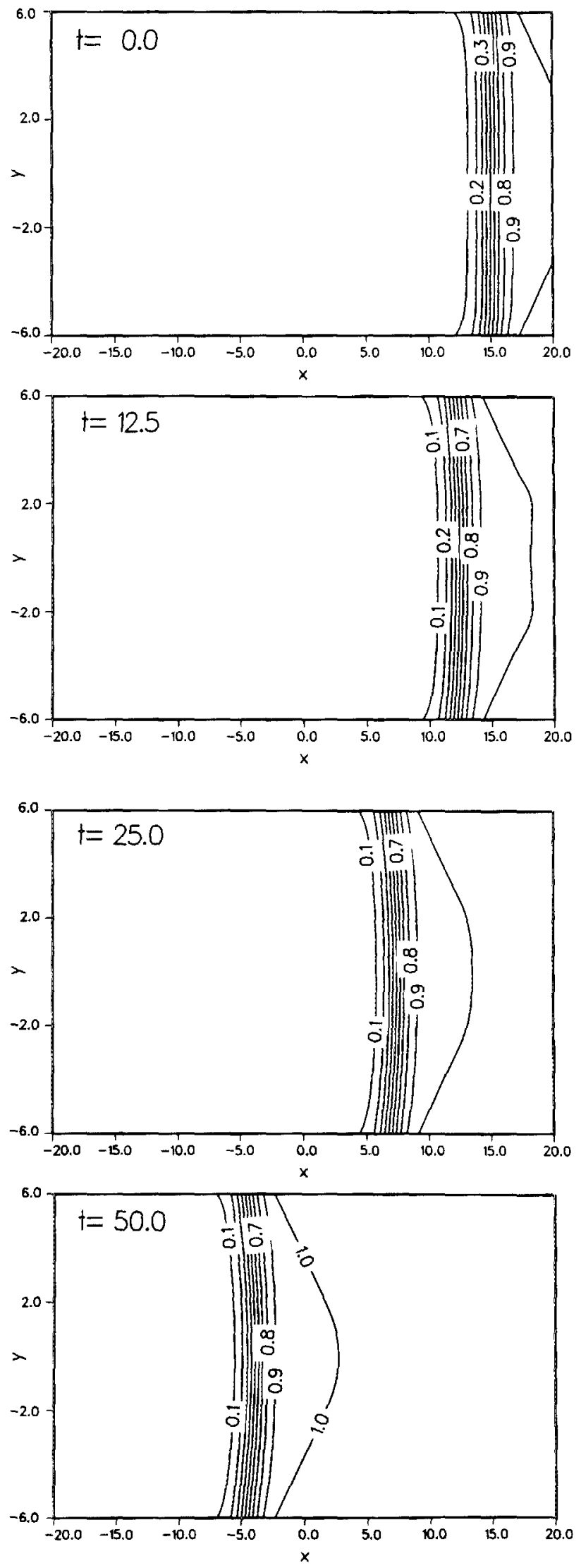

FIG. 2. Two-dimensional fluxon propagating in a rectangular Josephson junction. Parameter values $w=12, L=40, \alpha=0.1, p=0, x_{0}=15$, $u_{0}=0, \beta=0.4\left(\eta=2 / 3 \times 10^{-1}\right)$. Bias current in the direction of the $y$ axis. The results are displayed in terms of contour plots where $\varphi / 2 \pi$ equals multiples of 0.1 along the curves. The results are displayed for $t=0,12.5,25$, and 50. After transients, the fluxon is u shaped. The space step in the $x$ and $y$ direction is $h=1 / 32$. 

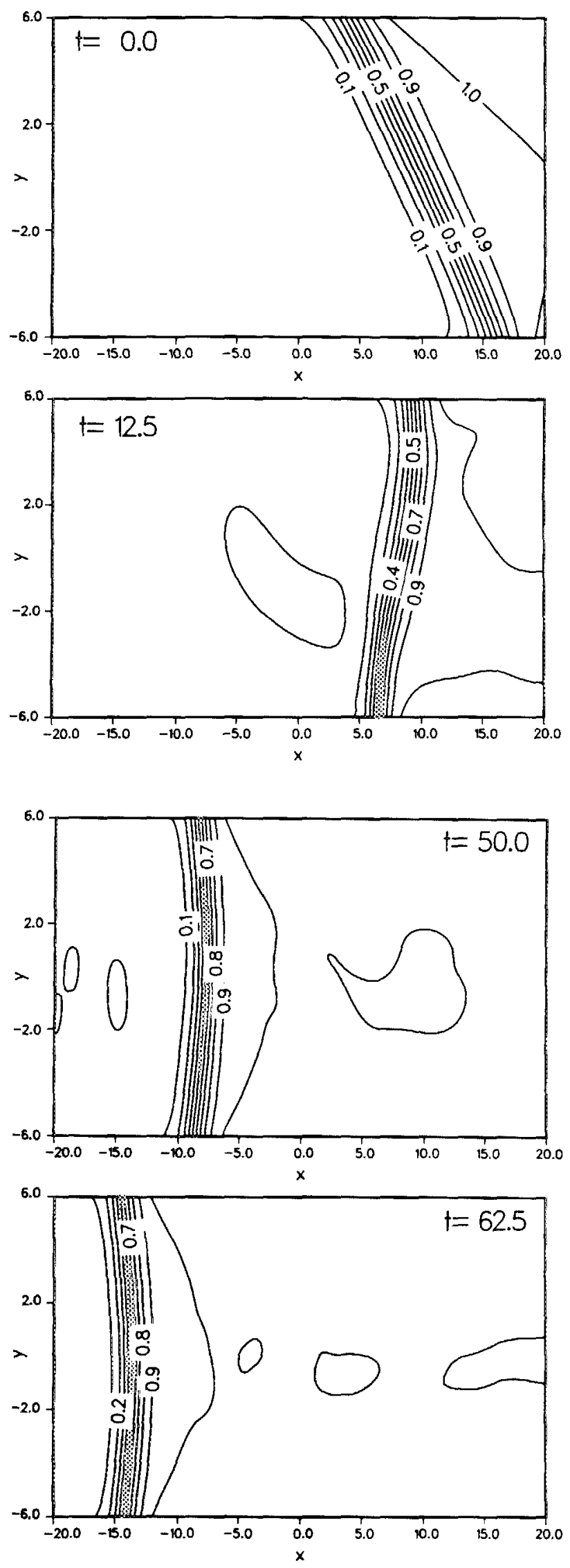

FIG. 3. Transient behavior of fluxon initially nonparallel to the $y$ axis. $\mathrm{Pa}$ rameter values as in Fig. 2 except for $p=1$. The results are displayed for $t=0,12.5,50$, and 62.5. Radiation is observed, but the $2 \pi$ kink eventually becomes parallel to the $y$ axis.

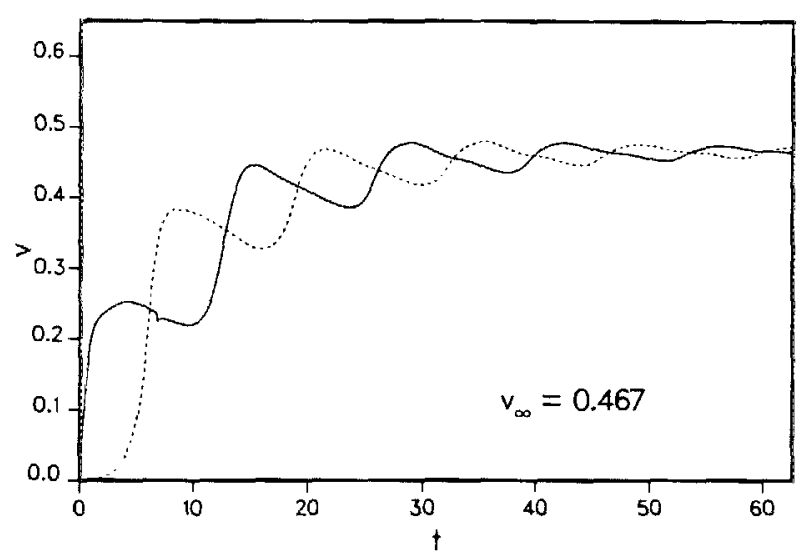

FIG. 4. Measured velocity of the fluxon as a function of time. Parameter values as in Fig. 2. The dashed curve represents the velocity measured at $y=0$, while the full curve represents the velocity measured at $y=6$. The time step in the calculation was $k=1 / 32$.

to two spatial dimensions. Denoting the restriction of $\varphi(x, y, t)$ to a square mesh by $\varphi_{m, l}^{n}=\varphi(m h, l h, n k)$ where $h=\sqrt{2} k$ (the stability limit for the linear equation) we get the following explicit formula as an approximation to Eq. (5)

$$
\begin{aligned}
\varphi_{m, l}^{n+1} & \\
= & (1-4 \sigma) \varphi_{m, l}^{n-1}+\sigma\left(\varphi_{m+1, l}^{n}+\varphi_{m, l-1}^{n}+\varphi_{m-1, l}^{n}\right. \\
& \left.+\varphi_{m, l+l}^{n}\right)-h^{2} \sigma \sin \left[\frac { 1 } { 4 } \left(\varphi_{m+1, l}^{n}+\varphi_{m, l-1}^{n}\right.\right. \\
& \left.\left.+\varphi_{m+1, l}^{n}+\varphi_{m, l+1}^{n}\right)\right] \\
\sigma= & {\left[2\left(1+\frac{\alpha h}{2 \sqrt{2}}\right)\right]^{-1} }
\end{aligned}
$$

The boundary conditions are treated by introducing an extra set of points outside the boundary to generate a second order approximation in the usual way. ${ }^{13}$ Throughout this paper we have used $k=1 / 32$.

In Fig. 2, the propagation of a two-dimensional fiuxon in a rectangular Josephson junction is shown. The parameter values in Eqs. (18) and (19) are $w=12, L=40, \alpha=0.1$, $p=0, x_{0}=15, u_{0}=0$, and $\beta=0.4$ corresponding to $\eta=2 /$ $3 \times 10^{-1}$. The bias current is flowing in the direction of the positive $y$ axis. The results are displayed in terms of contour plots where $\varphi / 2 \pi$ equals multiples of 0.1 along the curves. Snapshots are shown for $t=0,12.5,25$, and 37.5; after transients the fiuxon is seen to enter a mode where the $2 \pi$ change in the phase occurs along a line parallel to the $y$ axis-except near the boundaries where some distortion occurs. The explanation for this is that the energy of the fluxon is proportional to the "length" of the fluxon. Because of the dissipation, the energy in the junction is at a minimum, forcing the fiuxon to be parallel to the $y$ axis. In Fig. 3, we show the transient behavior of a fluxon which initially is nonparallel to the $y$ axis. The parameter values are as in Fig. 2 except for $p=1$. After a period with radiation-seen as closed loops in the figure-the fluxon straightens up and propagates parallel to the $y$ axis. For narrower junctions, the same behavior is observed.

In Fig. 4 we show the velocity of the fluxon displayed in Fig. 2 measured at $y=0$ and at the boundary $y=w / 2$. This 

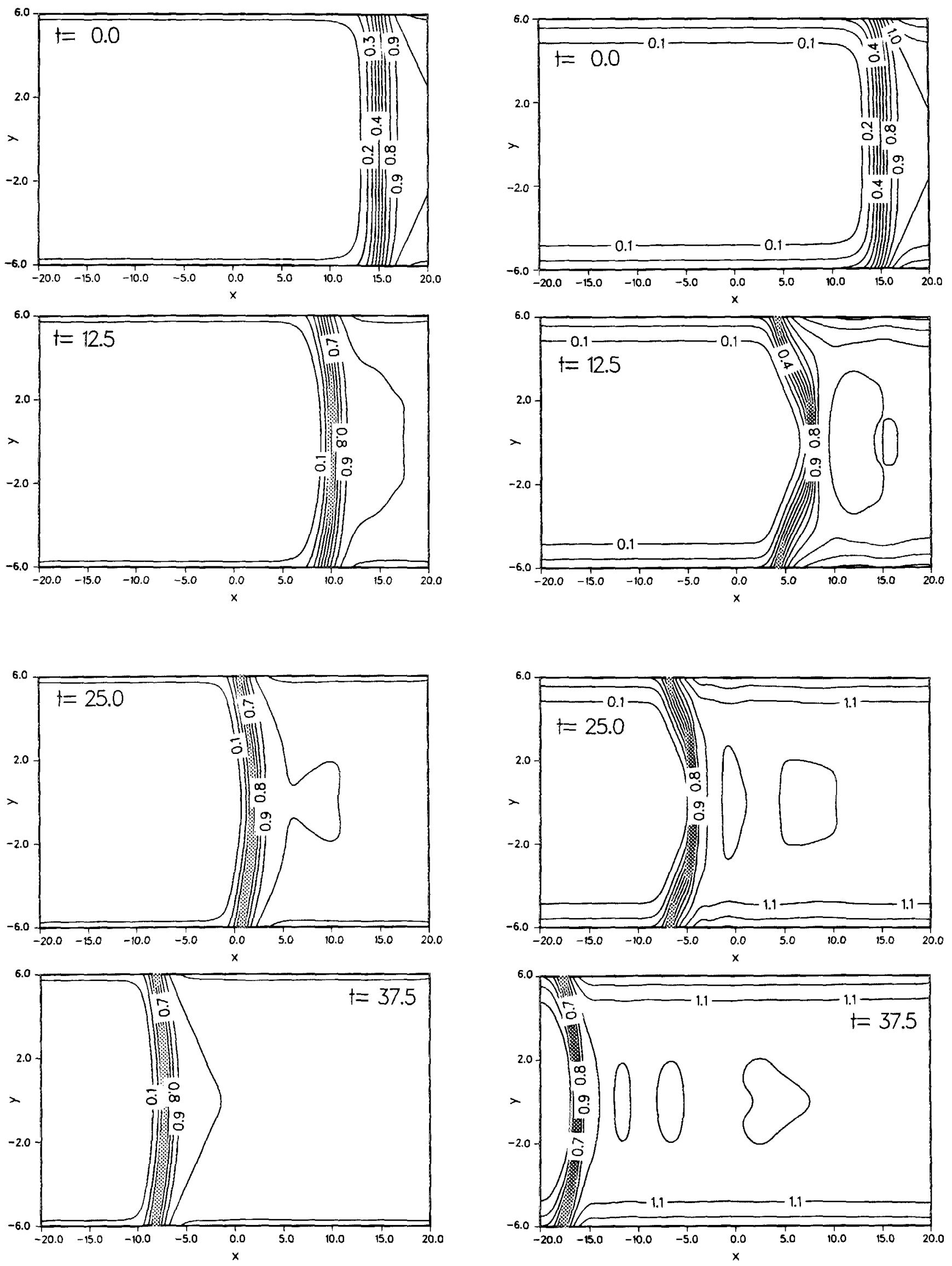

FIG. 5. Parameter values as in Fig. 2 except for $\beta=0.8\left(\eta=4 / 3 \times 10^{-1}\right)$. The effect of the boundary is visible. The results are displayed for $t=0$, $12.5,25$, and 37.5 .

FIG. 6. Parameter values as in Fig. 2 except for $\beta=1.6\left(\eta=8 / 3 \times 10^{-1}\right)$. The results are displayed for $t=0,12.5,25$, and 37.5 . 
is the velocity of the point where $\varphi=\pi$ obtained by means of a quadratic interpolation procedure. In contrast to the onedimensional case ${ }^{14}$ where the acceleration is Newtonian, we observe that the velocity oscillates while it increases. Further, the two velocities are out of phase. For junctions of smaller width, the period of the oscillation becomes smaller. This oscillatory behavior is due to the initial values. The velocities are approaching the stationary velocity $\approx 0.467 \pm 0.006$. The corresponding velocity from the one-dimensional model is 0.464 obtained from the expression

$$
\alpha u_{1 \mathrm{D}} \gamma\left(u_{1 \mathrm{D}}\right)=(\pi / 4) \eta\left(1-\eta^{2}\right)^{-1 / 8},
$$

which is a good fit of the velocity (agrees within $0.5 \%$ with the numerical experiments for $\eta<0.95$ ). This agreement is typical for smaller junctions and for $\beta<0.8$, for larger values of $\beta$ the determination of the velocity is difficult because of radiation created by the initial conditions. In Fig. 5 , the parameter values are as in Fig. 2 except for $\beta=0.8$. Again, the $2 \pi$ kink enters a stationary solution. The measured velocity is 0.73 while Eq. (21) gives 0.72 . Finally, in Fig. 6 we have raised the $\beta$ value to 1.6. The fluxon propagates in the usual way but radiates due to the boundary conditions. We have not in this investigation examined the stability of the fluxons to reflections, but mention that the fluxon in Fig. 2 annihilates while the fluxons in Figs. 5 and 6 survive the reflection.

We remark that a perturbation theory for fluxon propagation in the two-dimensional model can be developed using the usual approach ${ }^{14}$ but this leads to the same expression for the stationary velocity as obtained from the one-dimensional perturbation theory.

\section{CONCLUDHNG REMARKS}

In this paper, we have developed a model for two-dimensional Josephson junctions of the overlap type. This model reduces to the one-dimensional model for $\eta w^{2} / 8<1$. The equation derived for the critical current results in the critical current obtained for the one-dimensional model in the limit $w \rightarrow 0$. Numerical simulations of the propagation of a two-dimensional fiuxon show that the fluxon is perpendic- ular to the longer direction of the junction. This is due to energy dissipation. Comparisons between stationary fluxon velocities obtained from the two models show that only for very broad junctions the difference is important. Finally, we remark that the question of $2 \mathrm{D}$ fluxons stability to reflections is beyond the scope of this investigation but will be examined in the future.

\section{ACKNOWLEDGMENTS}

JCE would like to acknowledge the Royal Society of London for partial financial support during the period whilst this work was being carried out, and the US/UK Education Commission and the Carnegie Trust (Edinburgh) for further travel grants. OHO would like to thank the Danish Council for Scientific and Industrial Research (grant No. 863305.E-985) for financial support and the Center for Nonlinear Studies for its hospitality during his visit at Los Alamos. The work at Los Alamos was done under the auspices of the U. S. Department of Energy.

'G. Costabile, R. D. Parmentier, B. Savo, D. W. McLaughlin, and A. C. Scott, A ppl. Phys. Lett. 32, 587 (1978).

${ }^{2}$ R. D. Parmentier, in Solitons in Action, edited by K. Lonngren and, A. C. Scott, (Academic, New York, 1978), p. 173.

${ }^{3}$ D. W. McLaughlin and A. C. Scott, Phys. Rev. A 18, 1652 (1978).

${ }^{4}$ O. A. Levring, N. F. Pedersen, and M. R. Samuelsen, J. Appl. Phys. 54, 987 (1983).

${ }^{5}$ M. B. Fogel, S. E. Trullinger, A. R. Bishop, and J. A. Krumhansl, Phys. Rev. Lett. 36, 1411 (1976); 37, 314 (1976); Phys. Rev. B 15, 1578 (1977).

${ }^{\circ}$ P. S. Lomdahl, O. H. Sorensen, and P. L. Christiansen, Phys. Rev. B 25, 5737 (1982).

'P. S. Lomdahl, Ph.D thesis, The Technical University of Denmark, 1982, Report DCAMM-S20 (unpublished).

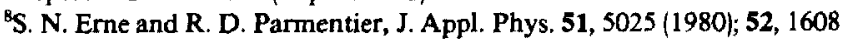
(1981).

'N. F. Pedersen and D. Welner, Phys. Rev. B 29, 2551 (1984).

${ }^{10} \mathrm{C}$. S. Owen and D. J. Scalapino, Phys. Rev, 164, 538 (1967).

' R. K. Dodd, J. C. Eilbeck, J. D. Gibbon, and H. C. Morris, Solitons and Nonlinear Wave Equations (Academic, London, 1982),p. 581.

${ }^{12}$ P. L. Christiansen and P. S. Lomdahl, Physica 2D, 482 (1981).

${ }^{13}$ A. R. Mitchell and D. F. Griffiths, The Finite Difference Method in Partial Differential Equations (Wiley, New York, 1980).

${ }^{14}$ O. H. Olsen and M. R. Samuelsen, Phys. Rev. B 28, 210 (1983). 